\title{
Combined radiotherapy and immunotherapy in urothelial bladder cancer: harnessing the full potential of the anti-tumor immune response
}

\author{
Mame Daro-Faye ${ }^{1}$. Wassim Kassouf ${ }^{2} \cdot$ Luis Souhami $^{3} \cdot$ Gautier Marcq $^{2,4,5} \cdot$ Fabio Cury $^{3} \cdot$ Tamim Niazi $^{6}$. \\ Paul Sargos ${ }^{6,7}$
}

Received: 8 July 2020 / Accepted: 3 September 2020 / Published online: 11 September 2020

(c) Springer-Verlag GmbH Germany, part of Springer Nature 2020

\begin{abstract}
Purpose Radiotherapy (RT), as part of trimodal therapy, is an attractive alternative treatment in patients with urothelial muscle-invasive bladder cancer (MIBC). There is accumulating evidence suggesting the immunomodulatory effects of RT and its potential synergy when combined with immunotherapy. The aim of this review was to report on the most recent advances on this combination, including the mechanisms of RT immunomodulation, practical approach to combining RT and immunotherapy, and ongoing clinical trials in bladder cancer.

Methods Using the PubMed database, we identified articles published between March 2004 and April 2020 on the combination of RT with immunotherapy in localized or metastatic MIBC. A search of the Clinicaltrials.gov and Clinicaltrialsregister. $\mathrm{eu} /$ retrieved ongoing clinical trials on the topic as well.

Results Combination of RT with immunotherapy leads to immunogenic cell death and an increase in immune markers thus leading to improved tumor control. For localized MIBC, there are safety concerns related to the use of concurrent immunotherapy with hypofractionated RT, thus neoadjuvant or adjuvant immunotherapy is preferred. In the metastatic setting, the combination of multi-site RT with SBRT-like doses ( $\geq 6$ Gy per fraction) and concurrent immunotherapy seems most efficacious at harnessing the abscopal effect. At least 25 clinical trials combining immunotherapy and RT in MIBC are currently ongoing and will answer pending questions on safety, efficacy, and practical considerations on RT scheduling, fractionation, and targets volumes.

Conclusion RT has the potential to synergize with immunotherapy to improve oncological outcomes in patient with localized or metastatic MIBC. Clinical trials results are eagerly awaited.
\end{abstract}

Keywords Radiotherapy · Radiation therapy · Immunotherapy · Immune checkpoint inhibitors · Urothelial carcinoma · Bladder cancer

Paul Sargos

p.sargos@bordeaux.unicancer.fr

1 Department of Radiation Oncology, McGill University Health Centre, McGill University, Montreal, QC, Canada

2 Department of Urology, McGill University Health Center, Montreal, QC, Canada

3 Fellow of the American Society for Radiation Oncology (FASTRO), Department of Radiation Oncology, McGill University Health Centre, Montreal, QC, Canada
4 Univ. Lille, Inserm, CHU Lille, U1189-ONCO-THAI-Laser Assisted Therapies and Immunotherapies for Oncology, 59000 Lille, France

5 Department of Urology, Claude Huriez Hospital, CHU Lille, 59000 Lille, France

6 Department of Radiation Oncology, Jewish General Hospital, Montreal, QC, Canada

7 Department of Radiation Oncology, Comprehensive Cancer Center, Institut Bergonie, 33076 Bordeaux Cedex, France 


\section{Introduction}

Trimodal therapy (TMT) is an attractive alternative treatment in patients with urothelial muscle-invasive bladder cancer (MIBC). TMT involves transurethral resection of the bladder tumor (TURBT), followed by radiotherapy (RT) and concurrent chemotherapy [1]. Its efficacy is comparable to that of the surgery in an appropriately selected population [2, 3]. Unfortunately, despite advances in these strategies, the 5-year overall survival (OS) for patients with non-metastatic T2-T4a disease remains around 50\% [4] while patients with metastatic disease have a 5-year OS of 13\% [5]. Moreover, local control rates with TMT range from 60 to $80 \%$ depending on disease stage and patient characteristics $[3,4]$. Thus, therapeutic innovations are urgently needed in the treatment of MIBC.

Immunotherapy has shown many promises in the past decade for the treatment of locally advanced and metastatic MIBC [6, 7]. Since 2016, five immune checkpoint inhibitors (ICIs) targeting the programmed-cell-death-1 (PD-1) and programmed-cell-death-ligand-1 (PD-L1) pathway have been approved by the FDA as second-line agents in the treatment of metastatic MIBC patients who have progressed on Cisplatin-based chemotherapy [8-12]. These agents showed a benefit in overall response rate (ORR) [8, 10-12] and in the case of Pembrolizumab, an OS benefit compared to chemotherapy [9]. Atezolizumab and Pembrolizumab are also approved as first line treatment in patients who are cisplatin-ineligible and whose tumors/infiltrating immune cells express PD-L1 $(\geq 5 \%)$ [13]. Despite these promising results, only about $20 \%$ of patients will respond to ICIs, although the majority of responders have a durable response $[8,14,15]$.

There is an accumulating body of evidence showing the immunomodulatory role of RT and its increased efficacy when combined with immunotherapy [16-19]. Thus, combining ICIs with RT could enhance both local and occult distant disease control in MIBC. The aim of this literature review is to report on the most recent advances on the topic, including the mechanisms of RT immunomodulation, practical approach to combine RT and ICIs and perspectives on ongoing clinical trials in metastatic and localized MIBC.

\section{Methods for evidence acquisition}

A literature search was performed in the PubMed database for articles on immunotherapy and RT in localized or metastatic MIBC. The following keywords were used in various algorithms: "radiotherapy," "radiation therapy," "immunotherapy," "immune checkpoint inhibitors," "urothelial carcinoma," "urothelial cancer," "bladder cancer." All sources published from March 2004 to April 2020 were included in the search. Original or review papers reporting on radiotherapy, immunotherapy, or the combination of, in localized or metastatic MIBC were included. Articles on upper urinary tract urothelial carcinomas and in language other than English or French were excluded. The articles were screened and further references relevant to the subject used. A search query was also done in Clinicaltrials.gov and Clinicaltrialsregister.eu to retrieve ongoing clinical trials on combined immunotherapy and RT in localized and/or metastatic MIBC.

\section{Radiotherapy and the immune system}

RT induces cell death by causing DNA damage, either directly through charged particles producing double strand breaks in DNA or indirectly by generating hydroxyl free radicals that will cause DNA damage, both leading to apoptotic cell death [20]. Apoptotic cell death has long been thought to be non-immunogenic; however, several pre-clinical studies have now shown that RT has both immunostimulatory and immunosuppressive properties through modulation of the tumor microenvironment (TME) (Table 1).

\section{Immune-stimulating effects of RT}

\section{Immunogenic cell death and modulation of the tumor microenvironment}

$\mathrm{RT}$ can induce a process known as immunogenic cell death by causing tumor cell stress and apoptosis, thus releasing tumor antigens in the TME $[17,21]$. RT has been shown to induce the expression and release of damage-associated molecular patterns (DAMPs) such as calreticulin, HSP70 and HMGB1 that are hallmarks of immunogenic cell death [16]. This process turns apoptotic cells into in-situ vaccines by releasing tumor antigens that are then presented to primed T-cells in the TME and draining lymph nodes [22]. Moreover, RT increases the expression of MHCI, pro-inflammatory cytokines as well as immune co-stimulatory molecules and adhesion molecules, thus facilitating CD8 + T-cell infiltration into the TME and priming [23]. Finally, RT can modulate the innate immune system by upregulating the complement pathway the co-stimulatory receptor NKG2D type II integral membrane protein leading to activation of NK cell-mediated responses [24]. 
Table 1 The effects of radiotherapy on the immune system

Immune-stimulating effects of radiotherapy

Immune-suppressing effects of radiotherapy

Induces immunogenic cell death:

Release of tumor antigens and DAMPs (calreticulin, HSP70, HMGB1)

Increased MHCI expression and APCs maturation

Increased CD8 + T-cell infiltration and tumor cell death

Increases:

Pro-inflammatory cytokines: interferon gamma, tumor necrosis factor- $\alpha$, type I interferons

Cos-stimulatory molecules

Adhesion molecules

Activates the innate immune system:

Upregulation of NKG2D type II

NK-cell activation

Radiation-induced lymphopenia (RIL):

Preferential depletion of CD4 $+\mathrm{T}$ cells and

B cells after RT

Abscopal effect:

$\uparrow$ tumor antigens $\rightarrow \uparrow \mathrm{APCs} \rightarrow \uparrow$ pro-inflammatory cytokines $\rightarrow \uparrow \mathrm{CD} 8+\mathrm{T}$ cells

Effects on infiltrating immune cells:

$\uparrow \mathrm{CD} 4+\mathrm{T}-\mathrm{reg}$ cells

$\uparrow$ MDSCs

Effects on immune cell surface markers:

$\uparrow$ PDL1 expression

$\uparrow$ CTLA4 expression on T-reg cells

$D A M P s$ damage-associated molecular patterns, $M H C I$ major histocompatibility complex class I, APC antigen presenting cell, $T$-reg $\mathrm{T}$ regulatory cells, MDSCs myeloid-derived suppressive cells, PDL1 programmed-cell death-ligand-1, CTLA4 cytotoxic T-lymphocyte-associated protein 4

\section{Radiation-induced abscopal effect}

The abscopal effect is the phenomenon by which systemic anti-tumor responses are observed outside of the primary site of local irradiation [25]. It has been described in a number of different malignancies, including metastatic renal cell carcinoma, melanoma and hepatocellular carcinoma among others [25]. The exact mechanisms of this phenomenon are not well known but are thought to be mediated by a systemic anti-tumor immune response [26]. Ionizing radiation is thought to increase tumor antigen presentation, subsequent activation of cytotoxic T-cells and increased production of a pro-inflammatory response [26]. Thus, combining RT with immunotherapy could provide an opportunity to boost abscopal response rates. In a mouse model of MIBC the combination of RT and anti-PD-L1 treatment resulted in significantly slower growth rate compared with RT alone in the irradiated xenograft tumors but also in the contralateral non-irradiated tumors, resulting in improved survival [27]. This abscopal effect has also been described when RT is combined with ICIs in several types of malignancies [28]. In a proof-of-principle clinical trial, Formenti et al. [19] showed an objective abscopal response in 9/34 patients (27\%) with solid metastatic cancers that received GM-CSF and irradiation to one metastatic lesion. In a randomized phase 1 trial, Sundahl et al. compared Pembrolizumab with sequential versus concomitant stereotactic body radiotherapy (SBRT) to the largest metastatic lesion in MIBC patients. There was a $44 \%$ ORR in non-irradiated metastatic sites when SBRT was given concomitantly vs. $0 \%$ when given sequentially, correlating with a median OS of 12.1 and 4.5 months, respectively [29]. Table 2 lists ongoing trials combining immunotherapy and RT in the metastatic setting, and the phase 2 trial NCT03601455 specifically studies the abscopal effect as a secondary objective.

\section{Immune-suppressing effects of RT}

\section{Radiation-induced lymphopenia}

Radiation-induced lymphopenia (RIL) is a well-recognized phenomenon that can develop in up to $70 \%$ of patients undergoing RT, especially when pelvic bony structures are irradiated [30]. RIL is characterized by acute preferential depletion of CD4+T-cells and B-cells [31]. In a study of 34 MIBC patients, RT caused a significant decline in the number of circulating lymphocytes for up to 5 years [32]. Interestingly, patients that were disease-free for five years had normalized lymphocyte counts to pretherapy levels within three years of RT whereas patients with recurrent or residual disease had significantly lower rate of RIL recovery [32]. Other studies have established RIL as a negative prognostic factor [33, 34]. Furthermore, lymphopenia can reduce the efficacy of ICIs [35, 36]. In a retrospective study of 167 patients treated with Nivolumab or Pembrolizumab, baseline and 3-month lymphopenia were associated with shorter PFS [35]. Interestingly, prior RT was the variable most strongly associated with persistent lymphopenia at 3-months and these patients had shorter PFS than patients whose lymphopenia recovered at 3-months [35]. High lymphocyte counts are also associated with better OS in ICIs-treated cancer patients $[33,37]$. Prospective studies are needed to firmly establish a causal relationship between RIL and clinical response to ICIs, but the available data suggests that RIL impairs ICIs efficacy.

\section{Effects on tumor infiltrating immune cells}

RT has also been shown to alter the profile of inhibitory immune cells infiltrating the TME. For instance, SBRT delivered to melanoma and breast cancer mice models 


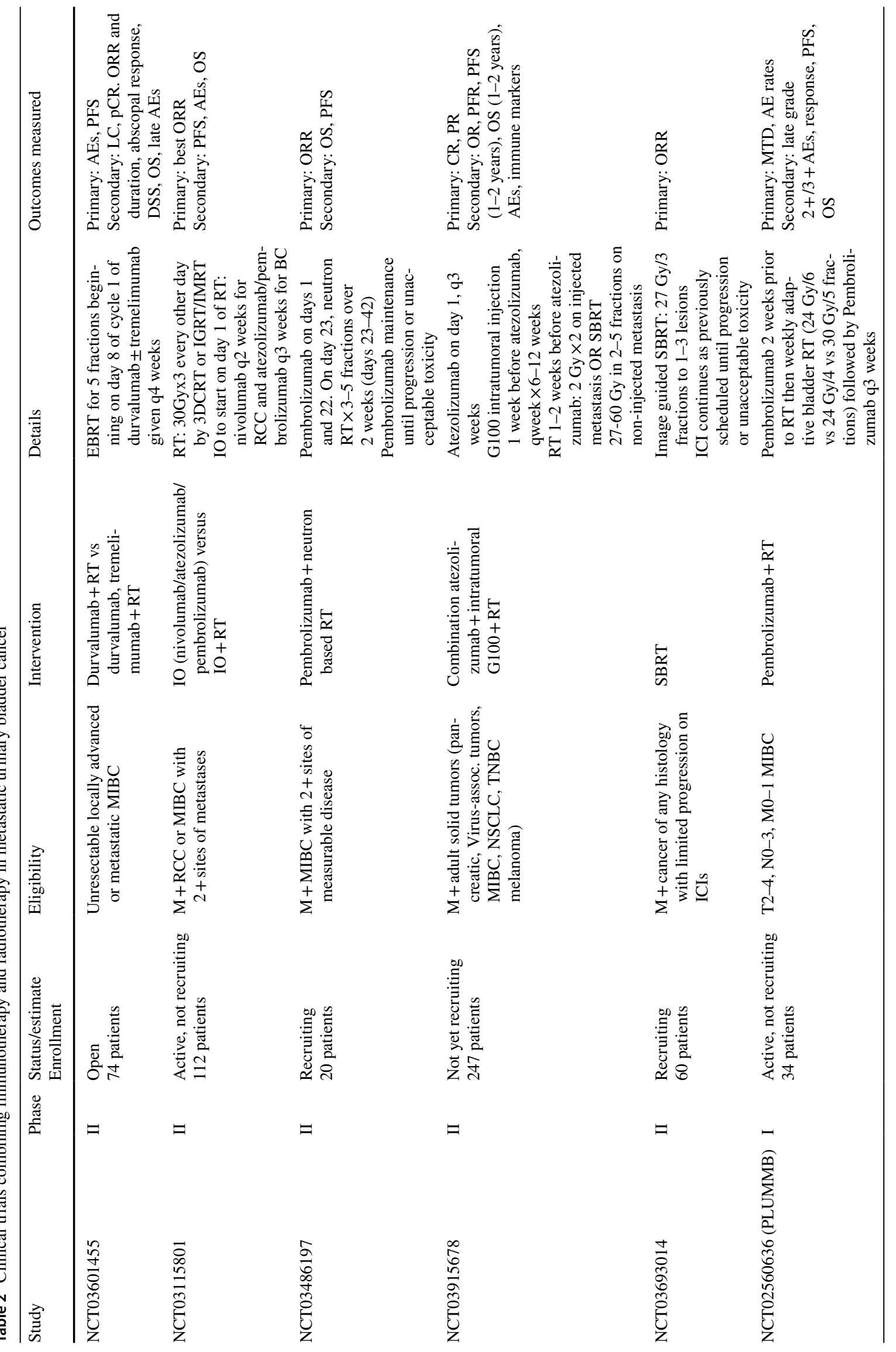


caused an increase in the proportion of $\mathrm{CD} 4+\mathrm{T}$ regulatory cells (T-reg) infiltrating the tumors [38]. This would a priori be detrimental to the anti-tumor response; however the increase in infiltrating T-reg cells was abrogated by addition of anti-PD-1 blockade, resulting in improved local control [38]. In their abscopal model on MIBC, Rompre-Brodeur et al. [27] showed that, compared to RT alone, mice treated with combined RT and ICIs had increased infiltration of cytotoxic T-cells, downregulation of immunosuppressive genes, and upregulation of T-cell activation markers. RT has also been shown to increase the infiltration and activation of myeloid-derived suppressive cells (MDSCs), which are known mediators of immunosuppression [39]. In another study of patients with oligometastatic solid tumors, treatment with concurrent SBRT and Sunitinib (but not SBRT alone) decreased the numbers of MDSC and T-reg cells, correlating with improved PFS and cause-specific survival [40].

\section{Effects on immune cell surface marker expression}

RT has also been shown to upregulate PD-L1 expression in several cancer types, notably in MIBC [41, 42]. RT upregulated the expression of PD-L1 in the human HT1197 and the murine MB49 MIBC cells and PD-L1 blockade in an orthotopic MB49 model was associated with tumor growth delay following irradiation [41]. Interestingly, when specimens from MIBC patients treated with chemoradiation were analyzed, high PD-L1 expression correlated with higher clinical stage, lower complete response rate and reduced diseasefree survival. There was also a positive correlation between PD-L1 overexpression and lymph nodes metastases or locoregional failure [41]. RT has also been shown to upregulate CTLA4 expression in T-reg cells [43].

\section{Practical considerations of combining RT with ICls in MIBC}

Although there is a large body of evidence supporting the synergistic effect of immunotherapy and RT, many questions remain on how to optimally combine these two modalities. Studies emphasize the importance of the sequencing, total dose, fractionation, and target volumes in harnessing this synergy.

\section{Sequencing}

Pre-clinical studies have explored the optimal sequencing of RT and immunotherapy in eliciting a synergistic immune response. In a colorectal cancer mouse model, Young et al. [44] showed that anti-CTLA4 was most effective when given 7 days prior to RT versus one day or one week after. Interestingly, anti-OXO was most effective 
when delivered one day post RT, highlighting the nuances in optimally combining RT with different immunotherapy regimen [44]. While some pre-clinical data show an increase PD-L1 expression and improved survival when RT was given concurrently with ICIs [41, 44], we were unable to show any difference in tumor growth rate inhibition when ICI was given either neoadjuvantly, concomitantly or adjuvantly with TMT (Tholomier et al. [45], in press). In contrast, in Sundahl et al. phase I trial of metastatic MIBC, ORR was $44.4 \%$ in the concomitant Pembrolizumab-SBRT vs. $0 \%$ in the sequential arm [29]. Ongoing clinical trials are evaluating combined immunotherapy with TMT in MIBC with various administration schedules: SWOG 1806 (NCT03775265) and KEYNOTE-992 (NCT04241185) are assessing concurrent chemoradiotherapy with atezolizumab or pembrolizumab, the CCTG BL13 study (NCT03768570) is evaluating adjuvant durvalumab after TMT, whereas the soon to open UK trial will examine neoadjuvant durvalumab followed by TMT.

\section{Doses and fractionation}

Different RT fractionation schemes and doses have been shown to have various immunomodulatory effects, either favoring immunostimulation or immunosuppression [46]. Suppressor T-cells are particularly radiosensitive whereas macrophages and regulatory $\mathrm{T}$-cells are more radioresistant $[23,46]$. This poses a challenge in normalizing response to treatment as RT doses and techniques can vary, ranging from delivering a single fraction to a metastatic deposit to a more protracted course of several weeks of conventionally or hypofractionated RT [47].

Pre-clinical studies have shown that dose per fraction greater than 6-8 Gy are required to produce an effective immunogenic response [22, 46, 48]. Furthermore, most of the studies describing an abscopal effect used SBRT or SBRT-like dose regimens (doses per fraction of $\geq 6$ Gy) [49]. The abscopal effect also seems to be related to the fractionation used. In many tumor types, a multi-fractionated regimen was superior to single dose regimens in decreasing tumor growth at non-irradiated sites [18, 48]. In a mouse model of breast and colon cancer, while all fractionations were effective at controlling the primary irradiated tumor, only the multi-fractionated regimens ( 8 Gyx 3 fractions or 6 Gyx5 fractions), but not the single dose regimen (20 Gy/1 fraction), synergized with antiCTLA4 to decrease distant tumor growth [48]. Specifically in bladder cancer mouse models, ICIs were more effective when combined with a $10 \mathrm{Gyx} 2$ [27] or $6.25 \mathrm{Gyx} 2$ [45] $\mathrm{RT}$ regimens than with a $10 \mathrm{Gyx} 1$ regimen. In the clinical setting, establishing the ideal RT dose and fractionation when combined with immunotherapy remains a challenge requiring further evaluation.

\section{RT volume and sites of disease}

RT could be delivered to the whole pelvis, to the bladder only, bone metastases or visceral metastases. In the context of TMT, it would be intuitive to treat the gross tumor disease \pm whole bladder. However, it remains unanswered whether pelvic elective nodal irradiation (ENI) could directly or indirectly affect the immune response. Preclinical data suggest that ENI can decrease the synergy between RT and ICIs likely by inhibiting the antigen-presentation process within the TME and in nearby draining lymph nodes. In a mouse model of colorectal or melanoma tumors treated with ICIs and $12 \mathrm{~Gy}$ in one fraction to the tumor \pm draining lymph nodes, ENI attenuated immune cell infiltration, chemokine expression and intratumoral antigen-specific CD8 + T-cells, thus decreasing the synergistic effect between RT and ICIs [50]. ENI also adversely affected survival when combined with ICIs [50]. Other studies have shown a strong correlation between the RT volume and RT-induced lymphopenia [30, 33]. Thus, to enhance the synergistic effect between RT and ICIs, target volumes not involving the pelvic lymph nodes may be preferable when combining RT with immunotherapy in the localized MIBC setting since indirect irradiation of bone marrow structures during ENI could induce lymphopenia. To our knowledge, there are no clinical trials currently addressing this question in MIBC.

In the metastatic setting, a relevant question is which metastatic site to irradiate if several are present. Most reported cases of the abscopal effect involved RT to visceral metastases [25], suggesting that visceral sites may be more immunogenic than osseous sites; although direct comparative studies are lacking. In a recent review, Brooks et al. [51] proposed the provocative idea that the singlesite irradiation abscopal approach should be abandoned to the benefit of comprehensive multi-sites irradiation when combing RT and ICIs. They formulated the hypothesis that irradiating multiple sites of disease reduces tumor burden while also increasing the likelihood of exposure and priming to the desired tumor-associated antigens. This would circumvent the inhibitory effects of the TME within each individual tissue bed, thus increasing the probability of activation of the anti-tumor immune process. Recent clinical trials studying ICIs in combination with multi-site irradiation support this hypothesis [52, 53]. Randomized trials comparing single to multi-site irradiation and stratifying patients with limited and extensive metastatic burden are needed. 


\section{Toxicities}

The adverse effects (AEs) associated with ICIs use (irAEs) and their management are well documented [54]. RT-related AEs are thought to be in part related to the immune system response, mostly through its effects on pro-inflammatory and fibrogenic cytokines [55]. There are concerns that the combination of RT and immunotherapy could lead to a cumulative toxicity profile. The safety considerations related to the combination of RT with ICIs in solid cancers have been reviewed elsewhere, with grade $\geq 3$ irAEs ranging from $7-31 \%$ across studies [56].

In the treatment of localized MIBC, acute AEs are mostly related to the combination of pelvic irradiation and concomitant chemotherapy. These, most commonly, include gastrointestinal (GI) and genito-urinary (GU) AEs. Acute GU AEs range from 4 to $21 \%$ across studies, whereas acute GI AEs range from 2 to $21 \%$ [1]. Late grade 3 pelvic toxicities occur in $2-7 \%$ of patients $[47,57]$. The use of hypofractionated RT can also lead to more GI toxicity in the TMT setting [47]. Recently, a phase I trial evaluated the safety of concomitant intravenous Atezolizumab (anti-PDL-1) in combination with hypofractionated TMT in patients with T2-T4aNOM0 MIBC (NCT03620435, Table 3). The study closed prematurely due to unacceptable grade 3 GI toxicity in $50 \%$ of the patients (Table 4) [58]. In addition, Tree et al. also reported unacceptable toxicity when using pembrolizumab and weekly hypofractionated RT for metastatic or locally advanced MIBC in the phase 1 PLUMMB trial (NCT02560636) [59]. The trial was stopped for amendment after two out of five patients developed grade 3 GU AEs and one experienced grade 4 rectal perforation (Table 4) [59]. Thus, caution should be taken when ICIs are given concurrently with hypofractionated RT. Since the sequencing of TMT and immunotherapy does not appear to affect efficacy in MIBC [45], and in light of acute toxicity concerns presented herein, currently we favor neoadjuvant or adjuvant immunotherapy.

Finally, it is important to note that the toxicity of combined ICIs and RT could be enhanced when chemotherapy is used in the context of TMT. In metastatic MIBC, RT delivered to visceral metastases, such as the lungs or liver could also yield different irAEs, including pneumonitis, hepatitis or hematologic toxicities $[54,56]$. Of course, the relative sensitivity of the irradiated organ and the technique/dose used will also impact on the toxicity profile.

\section{Perspectives}

Through its immunomodulatory capability, RT is being studied as a targeted therapy modality that can enhance systemic tumor control. A search of the ClinicalTrials.gov database as of March 31st, 2020 showed 615 ongoing clinical trials combining immunotherapy and RT, of which 24 are in MIBC patients. Several trials are looking into combined immunotherapy and RT in the locally advanced or metastatic setting (Table 2). Other studies are investigating combined ICIs and RT either in the neoadjuvant setting or concurrently with TMT as a bladder-preserving approach (Table 3 ). The use of ICIs as maintenance treatment after TMT is also being studied in patients that cannot undergo salvage radical cystectomy. These trials may improve outcomes in MIBC and broaden treatment options for patients, particularly for the non-negligible proportion who are too frail to either undergo chemotherapy or surgery.

\section{Conclusion}

The accumulating pre-clinical and clinical body of evidence reviewed in this article supports the hypothesis that through its cytotoxic and immunotherapy effects, RT has the potential to synergize with ICIs to improve oncological outcomes in patients with localized or metastatic MIBC. Increased toxicity might be challenging especially when combining ICIs and hypofractionated RT regimens. The many ongoing clinical trials on the subject will help answer many practical questions related to RT scheduling, dose, fractionation, and targets for RT. Undoubtedly, well-designed randomized trials are warranted in this newly developing field with special attention given to how effectively and accurately measure treatment response. 


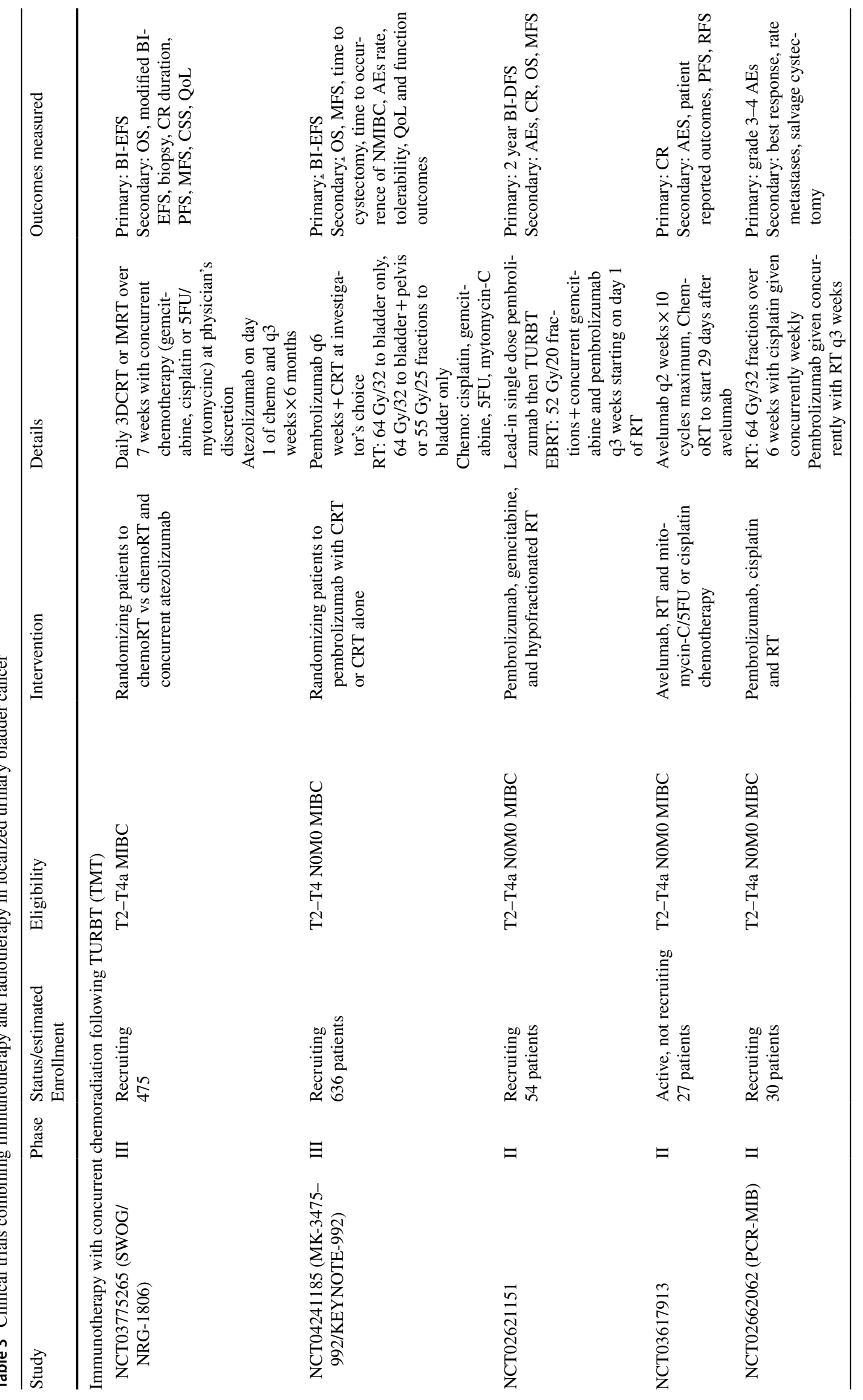




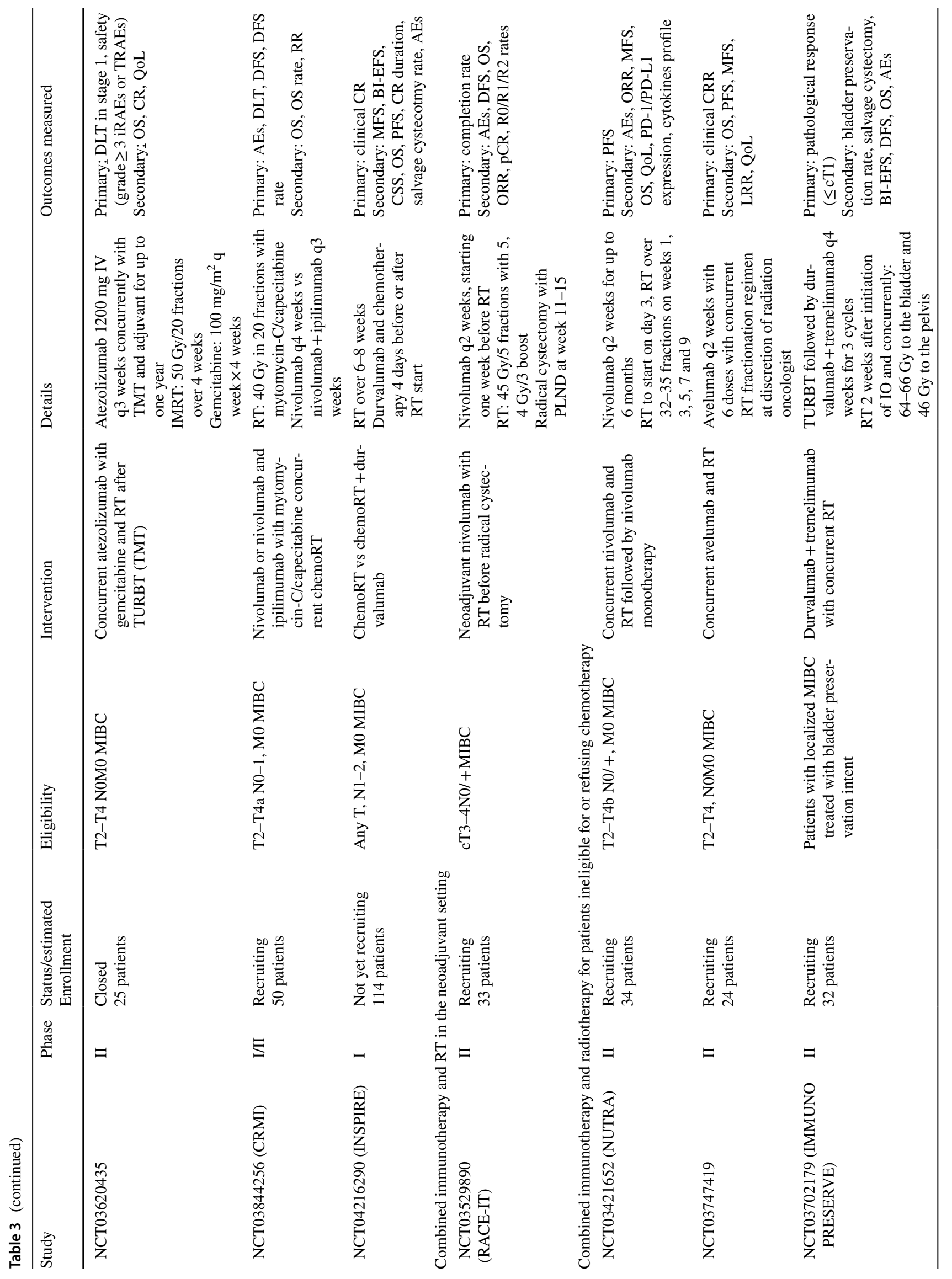




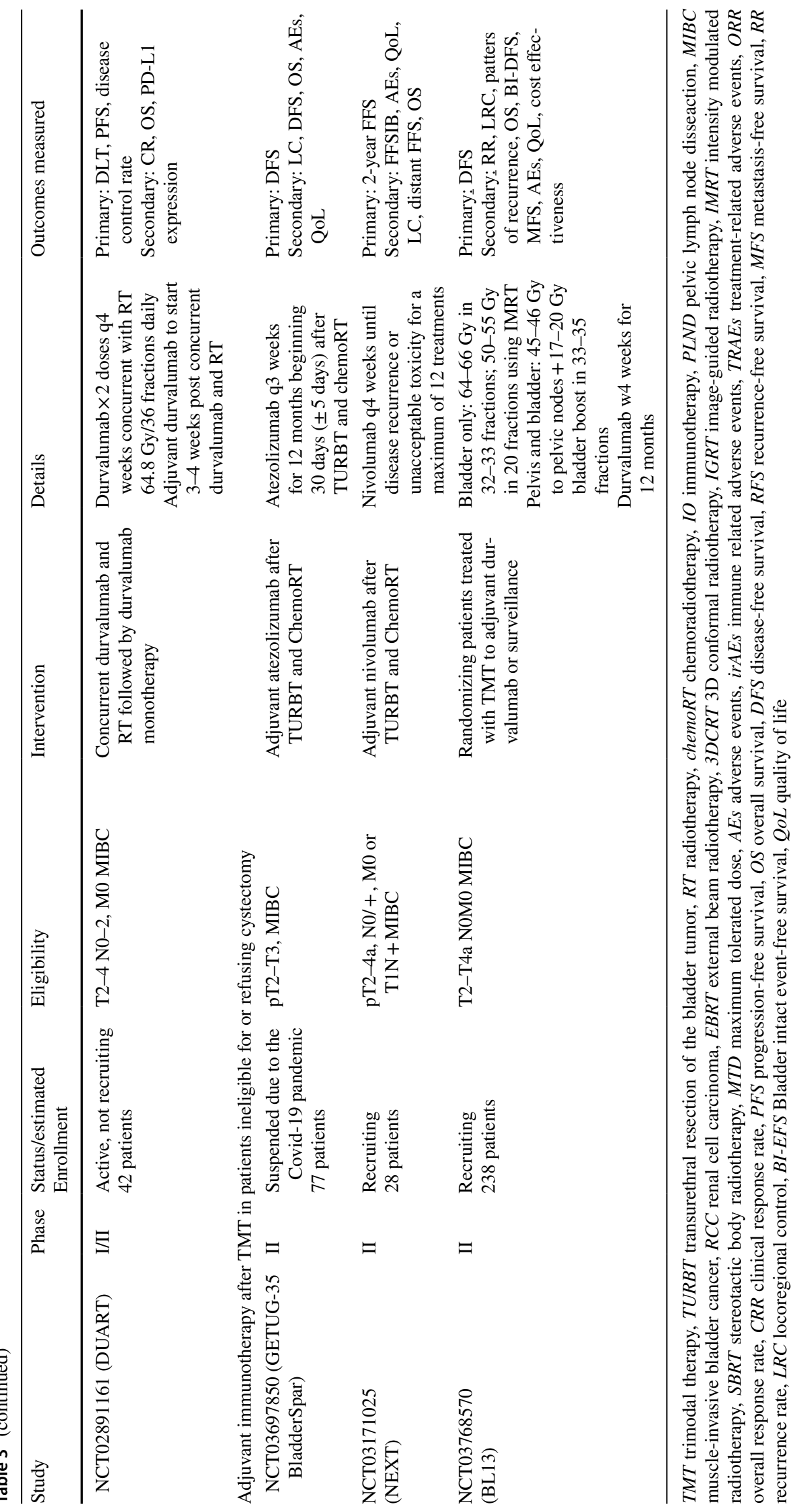


Table 4 Published studies on the safety of combined radiotherapy and immunotherapy in muscle-invasive bladder cancer

\begin{tabular}{|c|c|c|c|c|c|}
\hline Study & Study characteristics & Intervention & Safety outcomes & $\begin{array}{l}\text { Type of toxicities includ- } \\
\text { ing those that were not } \\
\text { DLT }(n)\end{array}$ & References \\
\hline $\begin{array}{l}\text { NCT02560636 } \\
\text { (PLUMMB } \\
\text { trial) }\end{array}$ & $\begin{array}{l}\text { Phase I trial involving } 5 \\
\text { patients in first cohort } \\
\text { with locally advanced or } \\
\text { metastatic MIBC (T2- } \\
\text { T4, N0-3, M0-1) }\end{array}$ & $\begin{array}{l}\text { Pembrolizumab } 2 \text { weeks } \\
\text { before weekly hypofrac- } \\
\text { tionated RT ( } 24 \text { Gy/6 } \\
\text { vs } 24 \mathrm{~Gy} / 4 \text { vs } 30 \mathrm{~Gy} / 5 \\
\text { fractions) }\end{array}$ & $\begin{array}{l}2 / 5 \text { patients met the } \\
\text { predefined definition of } \\
\text { dose-limiting toxicity } \\
\text { Trial was stopped and RT } \\
\text { doses reduced }\end{array}$ & $\begin{array}{l}\text { G4 bowel perforation }{ }^{\mathrm{a}}(1) \\
\text { G3 non-infective cystitis } \\
\text { (1) } \\
\text { G3 urinary tract/bladder } \\
\text { infection (2) } \\
\text { G3 hematuria (1) } \\
\text { G3 urinary pain (1) } \\
\text { G3 fatigue (1) } \\
\text { G2 urinary urgency, } \\
\text { incontinence (1) } \\
\text { G2 pain (1) } \\
\text { G2 anemia (1) }\end{array}$ & [59] \\
\hline NCT03620435 & $\begin{array}{l}\text { Phase I trial TMT in first } \\
\text { cohort of } 8 \text { patients with } \\
\text { T2-T4a N0M0 MIBC }\end{array}$ & $\begin{array}{l}\text { Concurrent atezolizumab } \\
\text { with gemcitabine and } \\
\text { hypofractionated RT } \\
\text { (50 Gy/20 fractions) } \\
\text { after TURBT (TMT) }\end{array}$ & $\begin{array}{l}\text { Study stopped after } 50 \% \\
\text { of patients experienced } \\
\text { grade } 3 \text { GI toxicities } \\
\text { despite atezolizumab } \\
\text { dose reduction. No grade } \\
4 \text { toxicity }\end{array}$ & $\begin{array}{l}\text { G3 colitis (3) } \\
\text { G3 proctitis (1) } \\
\text { G3 lymphopenia (1) } \\
\text { G3 neutropenia (1) }\end{array}$ & {$[58]$} \\
\hline
\end{tabular}

$D L T$ dose-limiting toxicity. $M I B C$ muscle-invasive bladder cancer, $R T$ radiotherapy, $G$ grade, $T U R B T$ transurethral resection of the bladder tumor, TMT trimodal therapy

${ }^{a}$ Happened outside of the DLT window, i.e. 11 weeks post completion of radiotherapy, thus considered at least subacute. All other toxicities are considered acute unless otherwise stated.

Author contributions MDF: Project development, Data Collection, Manuscript writing. PS: Project development, Manuscript editing, Supervision. WK: Manuscript editing. LS: Manuscript editing. GM: Manuscript editing. FC: Manuscript editing. TN: Manuscript editing.

\section{Compliance with ethical standards}

Conflict of interest All authors have no conflict of interest to disclose.

Research involving human participants and/or animals Not applicable.

Informed consent Not applicable.

\section{References}

1. Ploussard G et al (2014) Critical analysis of bladder sparing with trimodal therapy in muscle-invasive bladder cancer: a systematic review. Eur Urol 66(1):120-137

2. Efstathiou JA et al (2012) Long-term outcomes of selective bladder preservation by combined-modality therapy for invasive bladder cancer: the MGH experience. Eur Urol 61(4):705-711

3. James ND et al (2012) Radiotherapy with or without chemotherapy in muscle-invasive bladder cancer. N Engl J Med 366(16): 1477-1488

4. Giacalone NJ et al (2017) Long-term outcomes after bladderpreserving tri-modality therapy for patients with muscle-invasive bladder cancer: an updated analysis of the Massachusetts general hospital experience. Eur Urol 71(6):952-960
5. von der Maase $\mathrm{H}$ et al (2005) Long-term survival results of a randomized trial comparing gemcitabine plus cisplatin, with methotrexate, vinblastine, doxorubicin, plus cisplatin in patients with bladder cancer. J Clin Oncol 23(21):4602-4608

6. Lenfant $\mathrm{L}$ et al (2020) Current status and future directions of the use of novel immunotherapeutic agents in bladder cancer. Curr Opin Urol 30(3):428-440

7. Vera-Badillo FE, Tannock IF, Booth CM (2019) Immunotherapy for urothelial cancer: where are the randomized trials? J Clin Oncol 37(29):2587-2591

8. Rosenberg JE et al (2016) Atezolizumab in patients with locally advanced and metastatic urothelial carcinoma who have progressed following treatment with platinum-based chemotherapy: a single-arm, multicentre, phase 2 trial. Lancet 387(10031):1909-1920

9. Bellmunt J et al (2017) Pembrolizumab as second-line therapy for advanced urothelial carcinoma. N Engl J Med 376(11):1015-1026

10. Sharma $P$ et al (2016) Nivolumab monotherapy in recurrent metastatic urothelial carcinoma (CheckMate 032): a multicentre, open-label, two-stage, multi-arm, phase 1/2 trial. Lancet Oncol 17(11):1590-1598

11. Powles T et al (2017) Efficacy and safety of durvalumab in locally advanced or metastatic urothelial carcinoma: updated results from a phase 1/2 open-label study. JAMA Oncol 3(9):e172411

12. Patel MR et al (2018) Avelumab in metastatic urothelial carcinoma after platinum failure (JAVELIN solid tumor): pooled results from two expansion cohorts of an open-label, phase 1 trial. Lancet Oncol 19(1):51-64

13. Suzman DL et al (2019) FDA approval summary: atezolizumab or pembrolizumab for the treatment of patients with advanced urothelial carcinoma ineligible for cisplatin-containing chemotherapy. Oncologist 24(4):563-569 
14. Petrylak DP et al (2018) Atezolizumab (MPDL3280A) monotherapy for patients with metastatic urothelial cancer: long-term outcomes from a phase 1 study. JAMA Oncol 4(4):537-544

15. Fradet Y, Bellmunt J, Vaughn DJ, Lee JL, Fong L, Vogelzang NJ, Climent MA, Petrylak DP, Choueiri TK, Necchi A, Gerritsen W, Gurney H, Quinn DI, Culine S, Sternberg CN, Nam K, Frenkl TL, Perini RF, de Wit R, Bajorin DF (2019) Randomized phase III KEYNOTE-045 trial of pembrolizumab versus paclitaxel, docetaxel, or vinflunine in recurrent advanced urothelial cancer: results of $>2$ years of follow-up. Ann Oncol. 30(6):970-976. https://doi.org/10.1093/annonc/mdz127

16. Grassberger $\mathrm{C}$ et al (2019) Assessing the interactions between radiotherapy and antitumour immunity. Nat Rev Clin Oncol 16(12):729-745

17. Walshaw RC et al (2018) The anti-PD-1 era-an opportunity to enhance radiotherapy for patients with bladder cancer. Nat Rev Urol 15(4):251-259

18. Demaria $S$ et al (2005) Immune-mediated inhibition of metastases after treatment with local radiation and CTLA-4 blockade in a mouse model of breast cancer. Clin Cancer Res 11(2):728-734

19. Formenti $\mathrm{S}$ et al (2012) Pilot trial of radiation therapy and GM-CSF in metastatic cancer: abscopal responses. Int J Radiat Oncol Biol Phys 84(3):S178

20. Hall EJ, Giaccia AJ (2006) Radiobiology for the radiologist, vol 6. Lippincott Williams \& Wilkins, Philadelphia

21. Vanpouille-Box C et al (2015) In situ vaccination by radiotherapy to improve responses to anti-CTLA-4 treatment. Vaccine 33(51):7415-7422

22. Reits EA et al (2006) Radiation modulates the peptide repertoire, enhances MHC class I expression, and induces successful antitumor immunotherapy. J Exp Med 203(5):1259-1271

23. Thompson RF, Maity A (2014) Radiotherapy and the tumor microenvironment: mutual influence and clinical implications. Adv Exp Med Biol 772:147-165

24. Gasser $S$ et al (2005) The DNA damage pathway regulates innate immune system ligands of the NKG2D receptor. Nature 436(7054):1186-1190

25. Abuodeh Y, Venkat P, Kim S (2016) Systematic review of case reports on the abscopal effect. Curr Probl Cancer 40(1):25-37

26. Demaria $S$ et al (2004) Ionizing radiation inhibition of distant untreated tumors (abscopal effect) is immune mediated. Int J Radiat Oncol Biol Phys 58(3):862-870

27. Rompre-Brodeur A et al (2020) PD-1/PD-L1 immune checkpoint inhibition with radiation in bladder cancer: in situ and abscopal effects. Mol Cancer Ther 19(1):211-220

28. Ngwa W et al (2018) Using immunotherapy to boost the abscopal effect. Nat Rev Cancer 18(5):313-322

29. Sundahl N et al (2019) Randomized phase 1 trial of pembrolizumab with sequential versus concomitant stereotactic body radiotherapy in metastatic urothelial carcinoma. Eur Urol 75(5):707-711

30. Ellsworth SG (2018) Field size effects on the risk and severity of treatment-induced lymphopenia in patients undergoing radiation therapy for solid tumors. Adv Radiat Oncol 3(4):512-519

31. Campian JL et al (2017) Serial changes in lymphocyte subsets in patients with newly diagnosed high grade astrocytomas treated with standard radiation and temozolomide. J Neurooncol 135(2):343-351

32. O'Toole C, Unsgaard B (1979) Clinical status and rate of recovery of blood lymphocyte levels after radiotherapy for bladder cancer. Cancer Res 39(3):840-843

33. Tang $\mathrm{C}$ et al (2014) Lymphopenia association with gross tumor volume and lung V5 and its effects on non-small cell lung cancer patient outcomes. Int J Radiat Oncol Biol Phys 89(5):1084-1091
34. Liu LT et al (2018) The prognostic value of treatment-related lymphopenia in nasopharyngeal carcinoma patients. Cancer Res Treat 50(1):19-29

35. Diehl A et al (2017) Relationships between lymphocyte counts and treatment-related toxicities and clinical responses in patients with solid tumors treated with PD-1 checkpoint inhibitors. Oncotarget 8(69):114268

36. Ho WJ et al (2018) Association between pretreatment lymphocyte count and response to PD1 inhibitors in head and neck squamous cell carcinomas. J Immunother Cancer 6(1):84

37. Nakamura $Y$ et al (2016) Nivolumab for advanced melanoma: pretreatment prognostic factors and early outcome markers during therapy. Oncotarget 7(47):77404-77415

38. Sharabi AB et al (2015) Stereotactic radiation therapy augments antigen-specific PD-1-mediated antitumor immune responses via cross-presentation of tumor antigen. Cancer Immunol Res 3(4):345-355

39. Kumar V et al (2016) The nature of myeloid-derived suppressor cells in the tumor microenvironment. Trends Immunol 37(3):208-220

40. Chen HM et al (2015) Myeloid-derived suppressor cells as an immune parameter in patients with concurrent sunitinib and stereotactic body radiotherapy. Clin Cancer Res 21(18):4073-4085

41. Wu CT et al (2016) The role of PD-L1 in the radiation response and clinical outcome for bladder cancer. Sci Rep 6:19740

42. Deng L et al (2014) Irradiation and anti-PD-L1 treatment synergistically promote antitumor immunity in mice. J Clin Investig 124(2):687-695

43. Persa E et al (2015) The effect of ionizing radiation on regulatory $\mathrm{T}$ cells in health and disease. Cancer Lett 368(2):252-261

44. Young $\mathrm{KH}$ et al (2016) Optimizing timing of immunotherapy improves control of tumors by hypofractionated radiation therapy. PLoS ONE 11(6):e0157164

45. Tholomier C et al (2020) PD42-02 optimizing sequence of therapy and radiation delivery when combined with PD-L1 immunecheckpoint inhibition in bladder cancer. J Urol 203(Supplement 4):e827-e828

46. Schaue $\mathrm{D}$ et al (2012) Maximizing tumor immunity with fractionated radiation. Int J Radiat Oncol Biol Phys 83(4):1306-1310

47. Amestoy F et al (2019) Review of hypo-fractionated radiotherapy for localized muscle invasive bladder cancer. Crit Rev Oncol Hematol 142:76-85

48. Dewan MZ et al (2009) Fractionated but not single-dose radiotherapy induces an immune-mediated abscopal effect when combined with anti-CTLA-4 antibody. Clin Cancer Res 15(17):5379-5388

49. Brooks ED et al (2016) Stereotactic ablative radiation therapy combined with immunotherapy for solid tumors. Cancer J (Sudbury, Mass.) 22(4):257

50. Marciscano AE et al (2018) Elective nodal irradiation attenuates the combinatorial efficacy of stereotactic radiation therapy and immunotherapy. Clin Cancer Res 24(20):5058-5071

51. Brooks ED, Chang JY (2019) Time to abandon single-site irradiation for inducing abscopal effects. Nat Rev Clin Oncol 16(2):123-135

52. Doyen J et al (2017) Clinical outcomes of metastatic melanoma treated with checkpoint inhibitors and multisite radiotherapy. JAMA Dermatol 153(10):1056-1059

53. Bauml JM et al (2019) Pembrolizumab after completion of locally ablative therapy for oligometastatic non-small cell lung cancer: a phase 2 trial. JAMA Oncol 5:1283

54. Michot J et al (2016) Immune-related adverse events with immune checkpoint blockade: a comprehensive review. Eur J Cancer 54:139-148

55. Sridharan V et al (2016) Effects of definitive chemoradiation on circulating immunologic angiogenic cytokines in head and neck cancer patients. J Immunother Cancer 4(1):32 
56. Hwang WL et al (2018) Safety of combining radiotherapy with immune-checkpoint inhibition. Nat Rev Clin Oncol 15(8):477

57. Efstathiou JA et al (2009) Late pelvic toxicity after bladder-sparing therapy in patients with invasive bladder cancer: RTOG 89-03, 95-06, 97-06, 99-06. J Clin Oncol 27(25):4055

58. Marcq G et al (2020) MP61-08 A phase I/II trial of transurethral surgery followed by a combination of atezolizumab an ANTI-PDL-1 (MPDL3280A) with trimodal therapy in patients with muscle-invasive bladder cancer. J Urol 203(Supplement 4):e938-e938
59. Tree AC et al (2018) Dose-limiting urinary toxicity with pembrolizumab combined with weekly hypofractionated radiation therapy in bladder cancer. Int J Radiat Oncol Biol Phys 101(5):1168-1171

Publisher's Note Springer Nature remains neutral with regard to jurisdictional claims in published maps and institutional affiliations. 